\title{
Neonatal expression of RNA-binding protein IGF2BP3 regulates the human fetal-adult megakaryocyte transition
}

\author{
Kamaleldin E. Elagib, ${ }^{1}$ Chih-Huan Lu, ${ }^{1}$ Goar Mosoyan, ${ }^{2}$ Shadi Khalil, ${ }^{1}$ Ewelina Zasadzińska, ${ }^{3}$ Daniel R. Foltz,, ${ }^{3,4}$ Peter Balogh, \\ Alejandro A. Gru, ${ }^{1}$ Deborah A. Fuchs, ${ }^{5}$ Lisa M. Rimsza, ${ }^{6}$ Els Verhoeyen, ${ }^{7,8}$ Miriam Sansó, ${ }^{9}$ Robert P. Fisher, ${ }^{9}$ \\ Camelia Iancu-Rubin, ${ }^{2}$ and Adam N. Goldfarb ${ }^{1}$
}

\begin{abstract}
'Department of Pathology, University of Virginia School of Medicine, Charlottesville, Virginia, USA. ²Department of Medicine, Icahn School of Medicine at Mount Sinai, New York, New York, USA. ${ }^{3}$ Department of Biochemistry and Molecular Cenetics, University of Virginia, School of Medicine, Charlottesville, Virginia, USA. ${ }^{4}$ Department of Biochemistry and Molecular Genetics, Northwestern University Feinberg School of Medicine, Chicago, Illinois, USA. 'DDepartment of Pathology, University of Arizona College of Medicine, Tucson, Arizona, USA. 'Department of Laboratory Medicine and Pathology, Mayo Clinic Arizona, Scottsdale, Arizona, USA. ${ }^{7}$ Centre International de Recherche en Infectiologie (CIRI), Team EVIR, Inserm, U1111, Ecole Normale Supériere de Lyon, Université Lyon 1, CNRS, UMR5308, Lyon, France. ${ }^{8}$ Inserm U1065, Centre Méditerranéen de Médecine Moléculaire, Nice, France. ${ }^{9}$ Department of Oncological Sciences, Icahn School of Medicine at Mount Sinai, New York, New York, USA
\end{abstract}

Hematopoietic transitions that accompany fetal development, such as erythroid globin chain switching, play important roles in normal physiology and disease development. In the megakaryocyte lineage, human fetal progenitors do not execute the adult morphogenesis program of enlargement, polyploidization, and proplatelet formation. Although these defects decline with gestational stage, they remain sufficiently severe at birth to predispose newborns to thrombocytopenia. These defects may also contribute to inferior platelet recovery after cord blood stem cell transplantation and may underlie inefficient platelet production by megakaryocytes derived from pluripotent stem cells. In this study, comparison of neonatal versus adult human progenitors has identified a blockade in the specialized positive transcription elongation factor $b(P-T E F b)$ activation mechanism that is known to drive adult megakaryocyte morphogenesis. This blockade resulted from neonatal-specific expression of an oncofetal RNA-binding protein, ICF2BP3, which prevented the destabilization of the nuclear RNA 7SK, a process normally associated with adult megakaryocytic P-TEFb activation. Knockdown of ICF2BP3 sufficed to confer both phenotypic and molecular features of adult-type cells on neonatal megakaryocytes. Pharmacologic inhibition of IGF2BP3 expression via bromodomain and extraterminal domain (BET) inhibition also elicited adult features in neonatal megakaryocytes. These results identify ICF2BP3 as a human ontogenic master switch that restricts megakaryocyte development by modulating a lineage-specific P-TEFb activation mechanism, revealing potential strategies toward enhancing platelet production.

\section{Introduction}

During fetal development, i.e., ontogeny, mammalian hematopoiesis goes through multiple phases of reconfiguration. This reconfiguration encompasses both lineage output and phenotypic features within lineages. The lineage most dramatically affected by ontogenic stage consists of human megakaryocytes, long known to display distinct morphologies in the fetus and adult. In situ analysis of primary tissue has shown fetal megakaryocytes to be smaller and less polyploid than adult counterparts $(1,2)$. Size differences are even discernible between children less than 2 years of age and those more than 4 years of age, indicating postnatal persistence of ontogenic influence (3). Ontogenic stage also affects platelet function, with moderate to marked hyporesponsiveness observed in full-term and premature neonates, respectively $(4,5)$. These infantile properties

Conflict of interest: The authors have declared that no conflict of interest exists Submitted: June 3, 2016; Accepted: March 16, 2017.

Reference information: J Clin Invest. 2017;127(6):2365-2377.

https://doi.org/10.1172/JCl88936. have clinical significance, as they underlie the common clinical problems of neonatal thrombocytopenia and hemorrhage (6).

The molecular basis for ontogenic regulation of megakaryopoiesis remains unknown but consists of a cell-intrinsic mechanism initiating within multipotent progenitors. Thus, transplant recipients of neonatal, cord blood-derived hematopoietic stem and progenitor cells (CB HSPCs) have smaller megakaryocytes and slower platelet recovery compared with age-matched recipients of adult HSPCs, despite having equal megakaryocyte numbers (7). In ex vivo HSPC cultures, CB megakaryocytes show a greater than 10-fold enhancement in proliferation and markedly diminished morphogenesis compared with adult counterparts (8). The morphogenetic impairment correlates directly with diminished capacity for platelet release (9). Fetal liver (FL) HSPCs and embryonic stem cell progenitors have even more limited morphogenetic potential than CB HSPCs (10), indicating a graded influence of ontogenic stage. Importantly, the ontogenic influence on megakaryopoiesis, while affecting morphogenesis and proliferation, does not affect all aspects of the megakaryocyte differentiation program (8). 
A
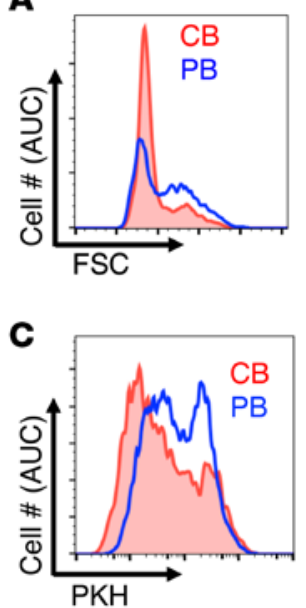
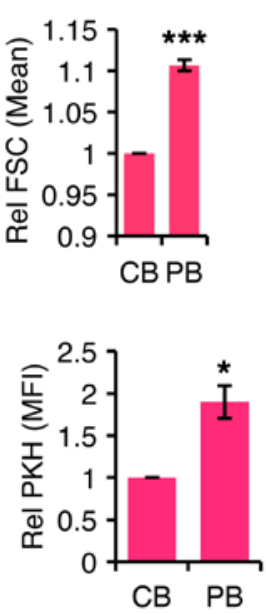

B
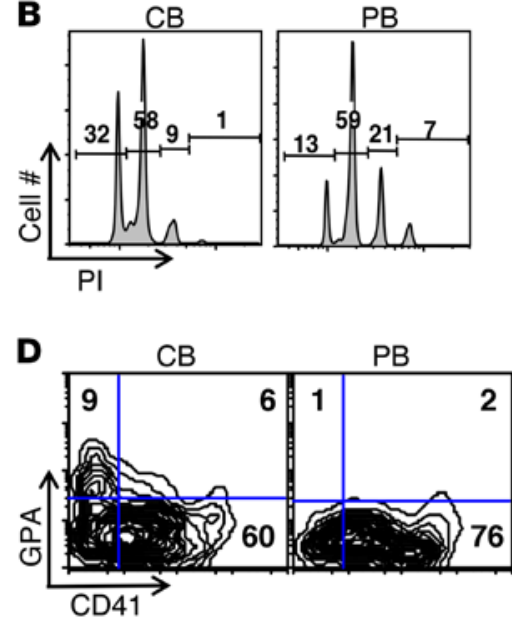
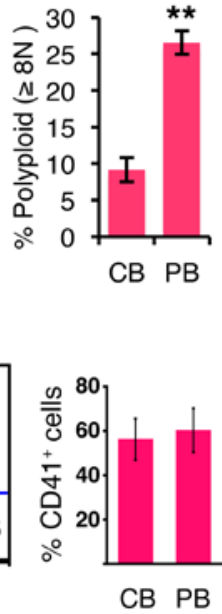
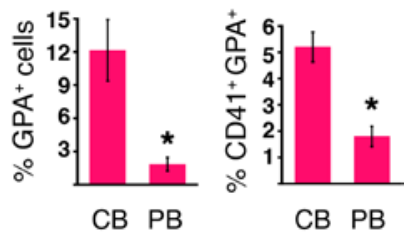

Figure 1. Neonatal megakaryocytes display decreased morphogenesis, enhanced proliferation, and incomplete erythroid silencing. (A-D) Purified adult PB and newborn CB progenitors cultured for 6 days in megakaryocytic medium were analyzed by flow cytometry for FSC in viable CD41+ cells (A), PI DNA staining in viable $C D 41^{+}$singlet cells (B), and $\mathrm{PKH}$ dye dilution in viable CD41+ cells (C). Graphs represent mean \pm SEM for 3 independent experiments. ${ }^{*} P<0.05 ;{ }^{*} P<0.01 ;{ }^{* *} P<0.005, t$ test. (D) Retention of erythroid antigen expression in neonatal megakaryocytes. Adult (PB) and newborn (CB) progenitors cultured for 6 days in megakaryocytic medium were analyzed by flow cytometry for erythroid (GPA) and megakaryocytic (CD41) markers on viable cells. Graphs represent mean \pm SEM for 3 independent experiments. ${ }^{*} P<0.05, t$ test. MFI, mean fluorescent intensity. See also Supplemental Figure 1.

Recently, megakaryocyte morphogenesis was found to depend on sustained, high-amplitude activation of the positive transcription elongation factor $\mathrm{b}$ (P-TEFb) kinase complex, consisting of CDK9 and cyclin T $(11,12)$. P-TEFb promotes RNA polymerase II (RNAPII) elongation on stimulus-responsive genes through phosphorylation of pausing factors $(5,6$-dichloro- $1-\beta-D-$ ribofuranosylbenzimidazole [DRB] sensitivity-inducing factor [DSIF] and negative elongation factor [NELF]) and the carboxy terminal domain of RNAPII large subunit (RNAPII CTD) (13). In most cells, a feedback loop maintains the majority of $\mathrm{P}-\mathrm{TEFb}$ sequestered in an inactive state within the 7SK small nuclear ribonucleo protein (snRNP) complex, which contains the kinase repressor hexamethylene bisacetamide inducible 1 (HEXIM1) scaffolded by the small nuclear RNA (snRNA) 7SK. This complex also contains the 7SK-stabilizing proteins methylphosphate capping enzyme (MePCE) and La ribonucleoprotein domain family member 7 (LARP7). Conventional activation occurs through stimulus-induced release of P-TEFb from the 7SK snRNP, promoting HEXIM1 transcription, which ultimately drives resequestration of P-TEFb (13). The megakaryocytic differentiation program, in contrast, employs a specialized activation pathway initiated by MePCE and LARP7 downregulation, leading to 7SK destruction and global, irreversible P-TEFb release (11). This mode of activation upregulates a cohort of cytoskeletal remodeling factors, such as megakaryoblastic leukemia (translocation) 1 (MKL1), filamin A (FLNA), and $\alpha$-actinin 1 (ACTN1), which function as morphogenesis effectors during differentiation $(11,14,15)$.

The current studies address whether the diminished morphogenesis of fetal megakaryocytes reflected impairment in this specialized program of P-TEFb activation. Supporting this concept, $\mathrm{CB}$ megakaryocytes failed to upregulate $\mathrm{P}-\mathrm{TEFb}$-dependent cytoskeletal remodeling factors and demonstrated deficiency in $\mathrm{P}-\mathrm{TEFb}$ activation. Despite lineage-appropriate downregulation of the 7SK stabilizing factors MePCE and LARP7, 7SK snRNA lev- els remained paradoxically elevated in CB megakaryocytes. These findings suggested the existence of a fetal-specific 7SK-stabilizing protein. Functional screening of candidate factors identified IGF2BP3 as a fetal-specific, 7SK-associated factor whose ectopic expression in adult HSPCs elicited fetal features. Knockdown of IGF2BP3 in CB HSPCs enabled adult-type megakaryopoiesis both phenotypically and in terms of P-TEFb activation, supporting a role as an ontogenic master switch within this lineage. Known regulation of IGF2BP3 by pluripotency circuits prompted a strategy to target its expression through bromodomain and extraterminal domain (BET) inhibition. This approach successfully downmodulated IGF2BP3 in CB megakaryocytes and elicited adult patterns of differentiation and $\mathrm{P}-\mathrm{TEFb}$ activation, indicating accessibility of this switch to exogenous manipulation.

\section{Results}

Neonatal megakaryocytes display decreased morphogenesis, enhanced proliferation, and incomplete erythroid silencing. To analyze molecular mechanisms involved in ontogenic regulation of megakaryopoiesis, we used unilineage culture of purified CD $34^{+}$HSPCs derived from normal neonatal umbilical CB or adult peripheral blood (PB). Gating strategies for the flow cytometric studies are illustrated in Supplemental Figure 1A (supplemental material available online with this article; https://doi.org/10.1172/JCI88936DS1). This system reliably modeled decreased enlargement (forward scatter [FSC] in Figure 1A), enhanced proliferation (dilution of PKH dye in Figure 1B), and decreased polyploidization (propidium iodide [PI] peaks in Figure 1C) of neonatal/fetal CD $41^{+}$megakaryocytes. Control studies showed no differences in initial PKH loading between sample types (Supplemental Figure 1B).

Woo et al. previously generated transcriptional profiles comparing fetal and adult murine megakaryocyte progenitors; in the top 122 fetal-upregulated transcripts, 7 erythroid genes were represented (16). Their results suggest an ontogenic influence on 
A
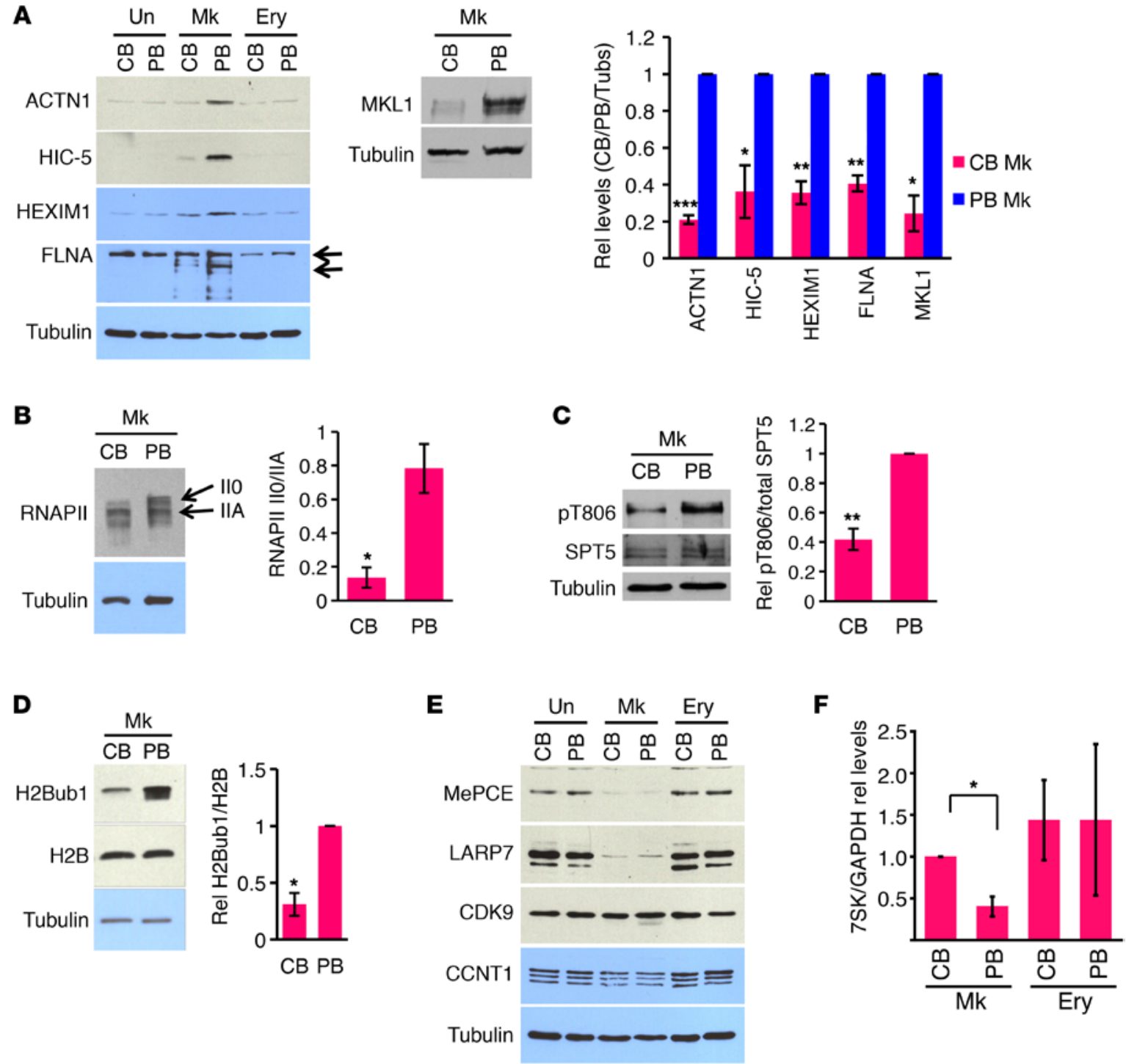

Figure 2. Neonatal progenitors fail to execute specialized megakaryocytic P-TEFb activation pathway. (A) Neonatal failure to upregulate megakaryocytic P-TEFb targets. Left panel: primary adult and neonatal progenitors, either undifferentiated (Un) or cultured 6 days in erythroid (Ery) or megakaryocytic (Mk) medium were immunoblotted for indicated factors. Top arrow, full-length FLNA; bottom arrow, approximately 190-kDa cleavage fragment. Right panel: densitometry comparing adult and neonatal megakaryocyte levels from 3 independent experiments conducted as in the right panel. Graphs show mean \pm SEM for signals normalized to tubulin, with PB values set at $1 .{ }^{*} P<0.05 ;{ }^{*} P<0.01 ;{ }^{* * *} P<0.005, t$ test. Rel, relative. (B-D) Evidence for diminished P-TEFb activation in neonatal versus adult megakaryocytes. Adult and neonatal progenitors cultured 6 days in megakaryocyte medium underwent IB for RNAPII subunit RPB1 (B), SPT5 phospho-threonine 806 (pT806) and total (SPT5) (C), and histone H2Bub1 and total H2B (D). IIO and IIA designate hyper- and hypophosphorylated forms for RNAPII, respectively. Graphs show mean \pm SEM for scanning densitometry values derived from 3 independent experiments. ${ }^{*} P<0.05 ;{ }^{* *} P<0.01, t$ test. (E) Neonatal megakaryocytes downregulate $75 K$-stabilizing factors to a degree similar to that of adult megakaryocytes. (F) Neonatal block in megakaryocytic downregulation of 7SK snRNA. 7SK levels relative to CB megakaryocytes in progenitors cultured as in $\mathbf{A}$. Graphs show mean \pm SEM of $7 S K$ normalized to GAPDH in 3 independent experiments. ${ }^{*} P<0.05, t$ test. Note that panels $\mathbf{A}$ and $\mathbf{E}$ as well as Figure $3 A$ and Supplemental Figure $4 \mathrm{~A}$ all derive from the same IB membrane and therefore share the same tubulin control. Note that panel $\mathbf{D}$ and Supplemental Figure 4B derive from the same IB membrane and share the same tubulin control. See also Supplemental Figures 2 and 3.

lineage consolidation of megakaryocytes emerging from bipotent megakaryocyte-erythroid progenitors, a notion further supported by the hybrid CD $41^{+}$glycophorin $\mathrm{A}^{+}(\mathrm{GPA})$ cells observed in pluripotent stem cell (PSC) hematopoiesis (17). We therefore determined whether human neonatal CB HSPCs manifested impairment in megakaryocytic lineage consolidation. In megakaryocytic unilineage medium, which lacks erythropoietin, adult PB HSPCs yielded essentially no erythroid $\mathrm{GPA}^{+}$cells, while CB
HSPCs consistently produced subpopulations of $\mathrm{CD} 41^{+} \mathrm{GPA} \mathrm{A}^{+}$ and $\mathrm{CD}^{-} \mathrm{GPA}^{+}$cells (Figure 1D), consistent with a diminished capacity for lineage consolidation.

Neonatal progenitors fail to execute the megakaryocytic P-TEFb activation pathway. In adult megakaryopoiesis, constitutive $\mathrm{P}$-TEFb activation drives upregulation of a cohort of cytoskeletal remodeling factors that function as critical effectors in morphogenesis (11). In neonatal megakaryopoiesis, in contrast, minimal 
A
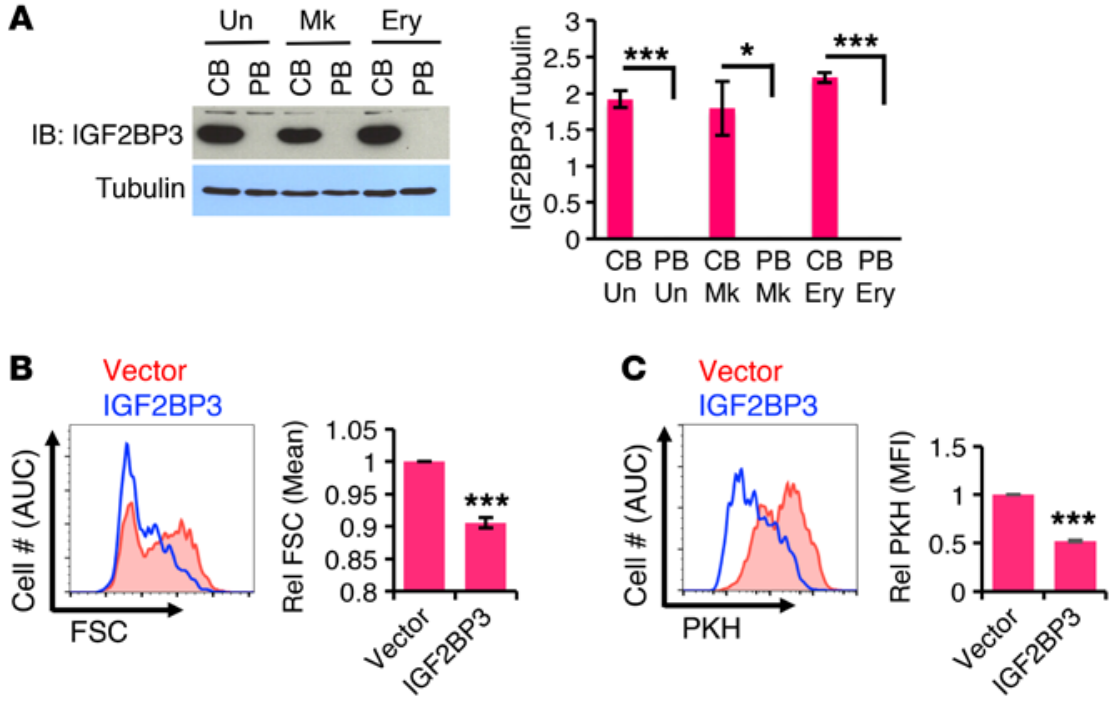

D

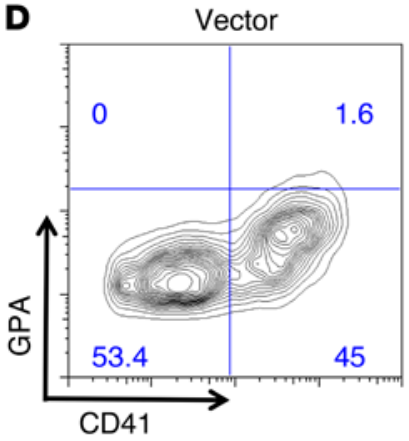

IGF2BP3

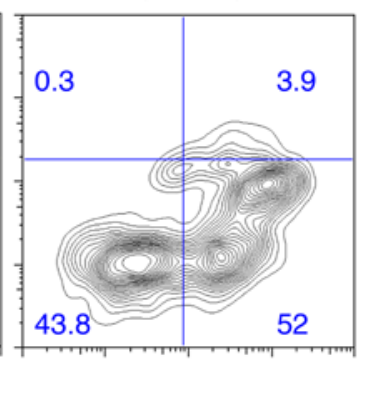

Figure 3. IGF2BP3 functions as a neonatal ontogenic determinant in megakaryocytes. (A) Specific and abundant expression of IGF2BP3 in neonatal hematopoietic progenitors. Experiments and quantitations were conducted as in Figure 2A. Graph depicts mean \pm SEM from 3 independent experiments. ${ }^{*} P<0.05$; ${ }^{* *} P<0.005, t$ test. (B) Block in adult megakaryocyte enlargement by ectopic IGF2BP3 expression. Adult progenitors transduced with retrovirus expressing GFP \pm IGF2BP3 underwent megakaryocyte culture for 6 days, followed by flow cytometry with gating on viable $\mathrm{CD} 41^{+} \mathrm{GFP}^{+}$cells. Graph depicts relative mean FSC \pm SEM from 3 independent experiments. ${ }^{* *} P<$ $0.005, t$ test. (C) Enhancement of adult megakaryocyte proliferation by ectopic IGF2BP3. Cells transduced and cultured as in B were subjected to PKH dye dilution assay, with quantitation by flow cytometry with gating on viable CD41+CFP+ cells. Graph depicts mean \pm SEM for PKH mean fluorescence intensity relative to control vector-transduced cells from 3 independent experiments. ${ }^{* *} P<0.005, t$ test. (D) Erythroid antigen induction in adult megakaryocyte by ectopic ICF2BP3. Cells transduced and cultured as in $\mathbf{B}$ underwent flow cytometry with gating on viable $\mathrm{GFP}^{+}$cells. Graph depicts mean \pm SEM for percentage of $C D 41^{+} \mathrm{CPA}^{+}$double positives from 3 independent experiments. ${ }^{*} P<0.05, t$ test. Note that panel $\mathbf{A}$, Figure $2 \mathrm{~A}$, Figure $2 \mathrm{E}$, and Supplemental Figure $4 \mathrm{~A}$ all derive from the same IB membrane and therefore share the same tubulin control. See also Supplemental Figures 4 and 5. upregulation of these factors occurred (Figure 2A). This defect, as well as a deficiency in HEXIM1 induction (Figure 2A), suggested impairment in P-TEFb activation. To further assess $\mathrm{P}-\mathrm{TEFb}$ signaling in neonatal megakaryopoiesis, additional downstream targets were examined. One such target, P-TEFb-mediated RNAPII CTD phosphorylation, is best reflected by the relative abundance of the more slowly migrating IIO isoform $(18,19)$. RNAPII phosphorylation on S2 does not provide a reliable readout, as CDK9 phosphorylates multiple positions on the CTD and multiple kinases phosphorylate the S2 position (20-23). Our studies consistently revealed a marked difference between neonatal and adult megakaryocytes in RNAPII isoform distribution (Figure 2B). In addition, a direct and specific P-TEFb substrate, SPT5 (18), showed 2.5-fold diminished phosphorylation in the neonatal versus adult megakaryocytes (Figure 2C). An epigenetic mark downstream of $\mathrm{P}-\mathrm{TEFb}$ activity consists of monoubiquitination of histone $\mathrm{H} 2 \mathrm{~B}$ on $\mathrm{K} 120$, H2Bub1 $(24,25)$. K120 is the amino acid position on the protein $\mathrm{H} 2 \mathrm{~B}$ which is modified by attachment of mono-ubiquitin to yield the modified protein H2Bub1. Notably, neonatal megakaryocytes displayed global deficiency in H2Bub1 as compared with adult cells (Figure 2D). Thus, assessment of multiple independent targets confirmed an ontogenically determined block in megakaryocytic P-TEFb activation.

As illustrated in Figure 2A, the P-TEFb target MKL1 showed markedly diminished expression in neonatal versus adult megakaryocytes cultured ex vivo. For this reason, and because MKL1 functions as a master regulator of megakaryocyte morphogenesis
(14), we further assessed its expression in human marrow biopsies by immunohistochemistry. Within the limited samples available, megakaryocytes from 2 adult stem cell transplant recipients showed strong staining for MKL1, while megakaryocytes from 3 CB stem cell transplant recipients showed negative to weak staining (Supplemental Table 1 and Supplemental Figure 2, A-D). In addition, megakaryocytes in marrow samples from 3 neonates (2 to 9 weeks of age) lacked detectable MKL1 (Supplemental Table 1).

Neonatal megakaryocytes downregulate 7SK-stabilizing factors, but fail to downregulate 7SK. The specialized pathway of $\mathrm{P}-\mathrm{TEFb}$ activation in adult megakaryocytes involves destabilization of 7SK snRNA through downregulation of its binding partners, the stabilizing factors LARP7 and MePCE (11). In this process, MePCE undergoes proteolysis due to megakaryocytic upregulation of calpain 2, which also cleaves the cytoskeletal remodeling factor FLNA. The appearance of FLNA cleavage fragments in both neonatal and adult megakaryocytes suggested intact calpain 2 regulation and function in the neonatal cells (Figure 2A). Indeed, both of the 7SK-stabilizing factors underwent full downregulation during neonatal megakaryopoiesis (Figure 2E and Supplemental Figure $3 \mathrm{~A})$. Numerous studies have documented tight coupling of 7SK snRNA levels with its stabilizing factors (26-30). However, the stabilizing factors' decline in neonatal megakaryocytes occurred in the absence of a proportionate drop in 7SK snRNA levels, which remained 2.5-fold higher than adult levels (Figure 2F and Supplemental Figure 3B). These findings suggest ontogenic differences in the composition and/or regulation of 7SK snRNP complexes. 

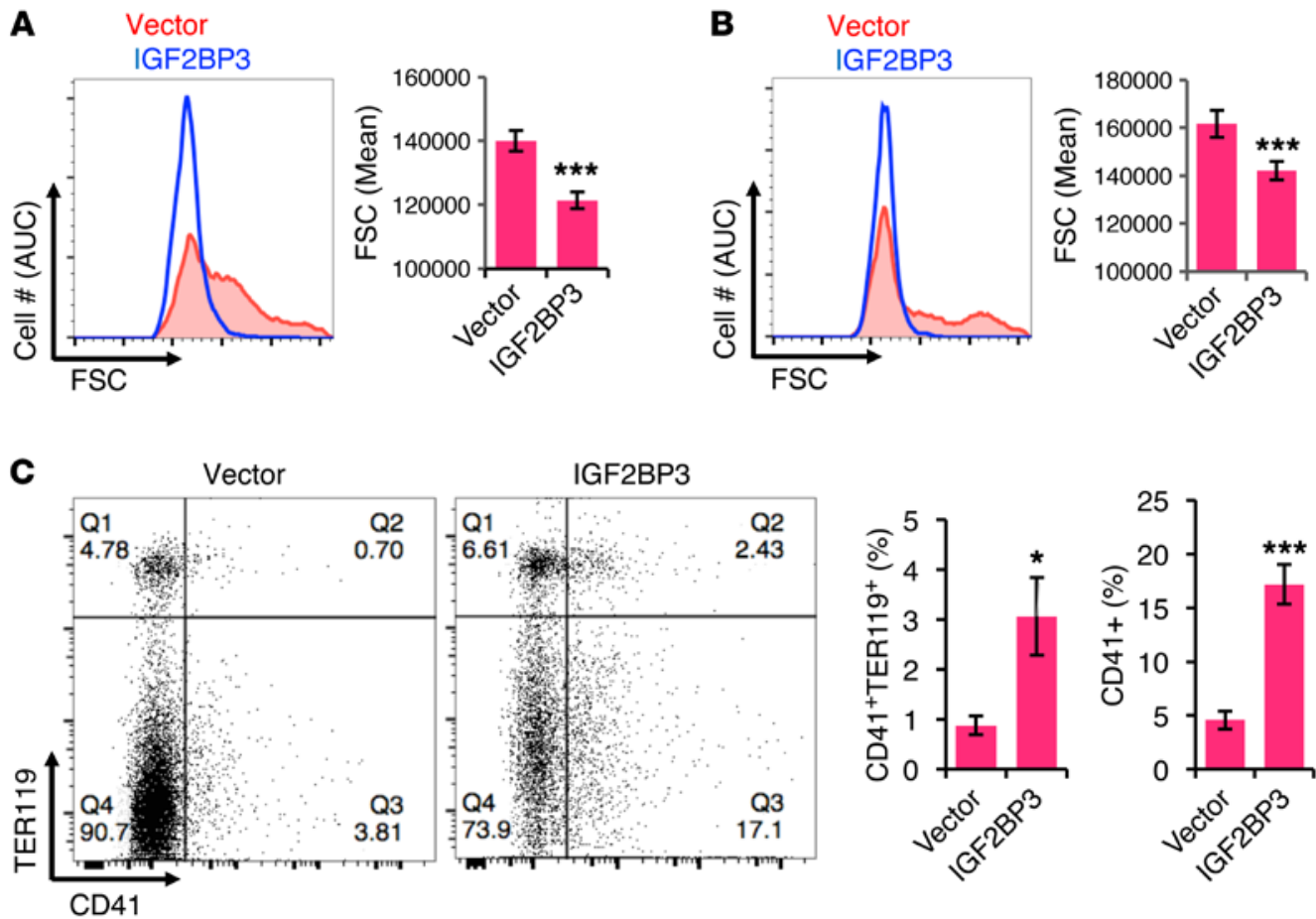

Figure 4. Enforced IGF2BP3 expression in vivo perturbs adult murine megakaryopoiesis. Adult murine marrow transduced with retrovirus expressing GFP \pm IGF2BP3 was transplanted into lethally irradiated mice. At 6 weeks after transplant, recipients were treated with murine TPO (2 mg/d IP $\times 3$ days). (A) Analysis of marrow megakaryocyte size (FSC) by flow cytometry, with gating on viable GFP+CD41+ cells. (B) Analysis of splenic megakaryocyte size (FSC) by flow cytometry, with gating on viable GFP+CD41+ cells. Graphs for $\mathbf{A}$ and $\mathbf{B}$ depict mean FSC \pm SEM. $n=6$ /group. ${ }^{* * *} P<0.005, t$ test. (C) Flow cytometry of marrow for CD41 and TER119 expression, with gating on viable GFP ${ }^{+}$cells. Graphs represent mean \pm SEM for percentages of CD41 ${ }^{+}$TER $119^{+}$double-positive cells and percentages of all CD41+ cells. $n=6$ /group. ${ }^{*} P<0.05 ;{ }^{* *} P<0.005, t$ test. See also Supplemental Figure 6.

An ontogenically regulated RNA-binding factor confers the neonatal phenotype. The relative stability of 7SK in neonatal megakaryocytes raised the possibility that fetal-specific RNA-binding factors might provide stabilizing functions in the absence of LARP7 and MePCE. To address this hypothesis, candidate factors underwent assessment first for ontogenic regulation and then for phenotypic influence on adult megakaryocytes. Candidates consisted of 7SK-associated or general RNA-binding factors that displayed upregulation in CB versus adult marrow HSPCs, based on available gene-expression profiles (31). One of the most compelling candidates consisted of IGF2BP3, a known oncofetal RNA-binding factor that participates in an interactome network containing 7SK-binding proteins $(32,33)$. IGF2BP3 protein levels markedly differed according to ontogenic stage, with highly abundant levels in neonatal hematopoietic cells, including megakaryocytes, and completely absent levels in adult counterparts (Figure 3A). An antibody recognizing both IGF2BP1 and IGF2BP2 showed their expression in both neonatal and adult megakaryocytes, although with differences in isoform patterns (Supplemental Figure 4A). Fetal-selective protein expression in megakaryocytes was also observed for the candidates LIN28B and HMGA1 (Supplemental Figure 4, B and C). Ectopic expression in adult megakaryocytes of IGF2BP3, but not of LIN28B or HMGA1, blocked cellular enlargement (Figure 3B and Supplemental Figure 4D). Furthermore, IGF2BP3 expression caused adult megakaryocytes to undergo additional rounds of cell division, as reflected by enhanced PKH dye dilution, and promoted expression of the erythroid marker GPA (Figure 3, C and D; see
Supplemental Figure 4E for initial PKH dye loading). These effects were specific for adult progenitors, as similar transduction of neonatal progenitors minimally altered their megakaryocytic phenotype (Supplemental Figure 5). Thus, IGF2BP3 fulfills multiple criteria for a megakaryocytic ontogenic regulator.

To confirm these findings using an in vivo model system, IGF2BP3 expression was enforced in adult murine marrow by retroviral transduction followed by transplantation. Prior studies in mice have shown Igf $2 b p 3$ transcript levels to be much higher (4- to 16-fold) in fetal versus adult hematopoietic stem cells and megakaryocytic progenitors (MkPs) $(16,34)$. Animals receiving IGF2BP3-transduced marrow retained transgene expression at 6 weeks after transplant. The chimeras were studied under steadystate conditions and after stimulation of megakaryopoiesis with thrombopoietin (TPO). Under both conditions, enforcement of IGF2BP3 interfered with megakaryocytic enlargement, reflected by diminished FSC in gated $\mathrm{GFP}^{+} \mathrm{CD} 41^{+}$cells from marrow and spleen (Figure 4, A and B, and Supplemental Figure 6A). In addition, ectopic IGF2BP3 increased the marrow frequency of CD41 ${ }^{+}$ cells and of $\mathrm{CD}_{4} 1^{+} \mathrm{Ter} 119^{+}$double-positive cells (Figures $4 \mathrm{C}$ and Supplemental Figure 6B). These latter findings were consistent with the enhanced megakaryocytic proliferation and erythroid antigen retention seen with ectopic IGF2BP3 in adult human progenitors (see Figure 3, C and D).

IGF2BP3 participates in $7 S K$ snRNP and contributes to 7SK stabilization. Prior studies have shown a capacity for IGF2BP3, but not IGF2BP1 or IGF2BP2, to undergo nuclear localization (35). In our 
A
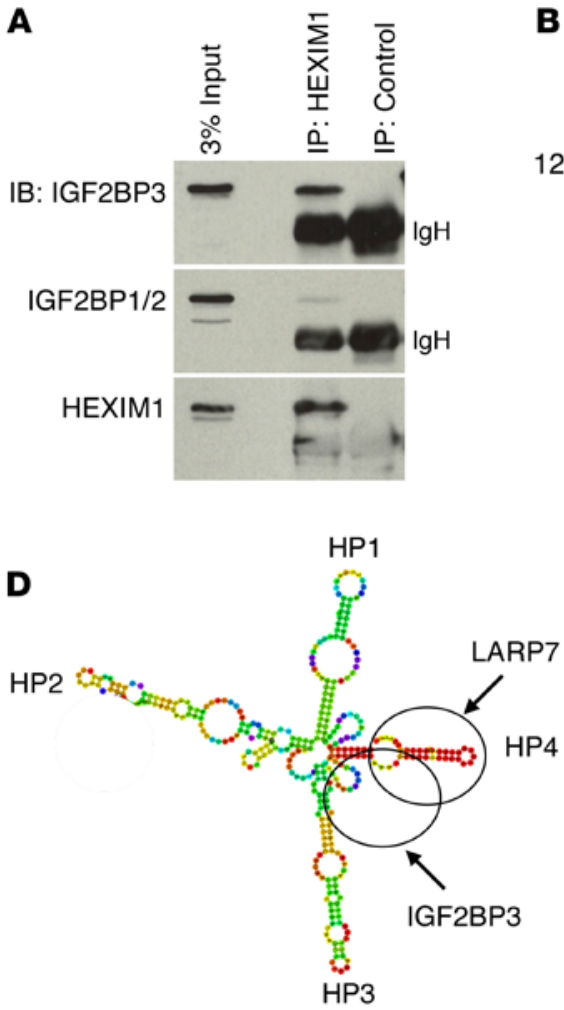

B

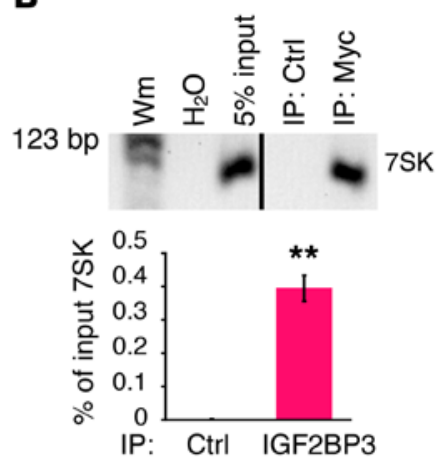

C

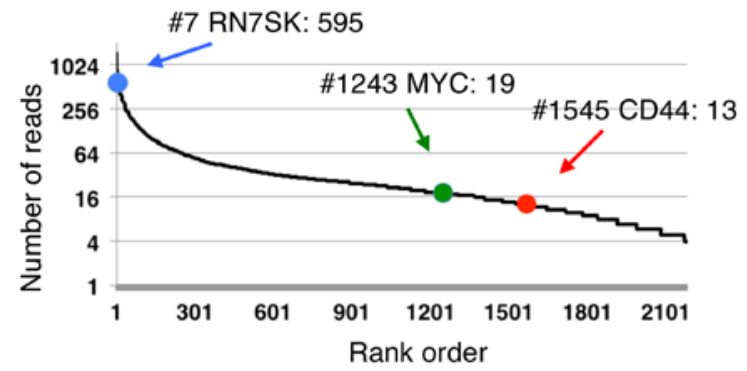

E

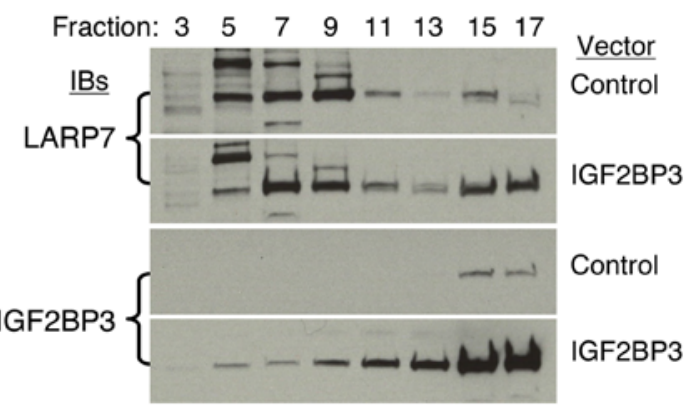

$\mathbf{F}$

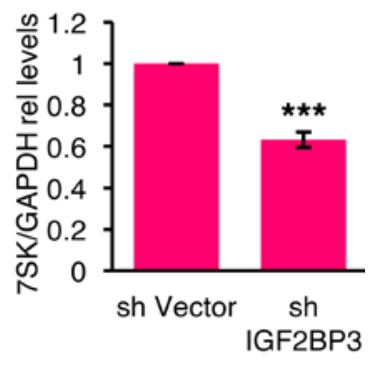

Figure 5. IGF2BP3 interacts with and regulates 7SK snRNP. (A) Interaction of endogenous IGF2BP3 and HEXIM1. K562 cellular extracts underwent IP with anti-HEXIM1 or control antibody followed by IB analysis as indicated. (B) Binding of 7SK snRNA by IGF2BP3. Extracts from 293 transfectants expressing Myc epitope-tagged ICF2BP3 underwent IP with anti-Myc or control antibody followed by reverse-transcriptase PCR (RT-PCR) with gel and real-time qPCR assessment. Graph depicts mean qPCR signal relative to input from 3 independent experiments. ${ }^{* *} P<0.01, t$ test. Line in gel indicates position of cropping of empty lanes. (C) Interaction of endogenous IGF2BP3 and 7SK. IGF2BP3 iCLIP-seq hits of Palanichamy et al. (36) from RS4;11 cells were arranged in order of read frequency. Rank and read counts are shown for 7SK, MYC, and CD44. (D) IGF2BP3 binds 7SK within HP4, a stability target for LARP7. Human 7SK folding was modeled using RNAfold server (ViennaRNA Web Services, Universitat Wien, Vienna, Austria). IGF2BP3 contact regions from iCLIP peak and known LARP7-binding region are depicted by ovals. (E) Alteration of LARP7 complexes by ICF2BP3. Extracts from 293 cells transfected with control or IGF2BP3 expression vector underwent fractionation by glycerol gradient sedimentation, with IB analysis of representative fractions. (F) ICF2BP3 contribution to 7SK stability in neonatal megakaryocytes. Neonatal progenitors transduced with lentiviral shRNA (sh) vectors underwent megakaryocyte culture and qRT-PCR as in Figure 2F. Graphs represent mean \pm SEM of 7SK normalized to GAPDH in 3 independent experiments. ${ }^{* *} P<0.005, t$ test. See also Supplemental Figure 7.

studies, neonatal megakaryocytes displayed both nuclear and cytoplasmic IGF2BP3 localization, as assessed by confocal immunofluorescence microscopy and subcellular fractionation (Supplemental Figure 7, A and B). Association with the 7SK snRNP was supported by endogenous co-IP of IGF2BP3 with HEXIM1 (Figure 5A and Supplemental Figure 7C). In addition, 7SK snRNA coprecipitated with epitope-tagged IGF2BP3 in 293 transfectants (Figure 5B). This experiment employed ectopic expression of epitope-tagged IGF2BP3 in 293 cells because of the lack of available antibodies suitable for native IP and because of the minimal endogenous IGF2BP3 in 293 cells. Mining of iCLIP-seq data examining endogenous IGF2BP3 interactions (36) revealed 7SK to be one of the top targets (7th out of 2185), far ahead of the canonical targets MYC and CD44 (Figure 5C). Notably, the iCLIP-seq data mapped IGF2BP3 binding to 7SK hairpin 4 (HP4), adjacent to a stabilizing binding site for LARP7 (37) (Figure 5D).

To further assess effects on the 7SK snRNP, 293 transfectants underwent biophysical characterization of LARP7, a dedicated component of this complex (29). Enforced IGF2BP3 expression redistributed LARP7 into high-molecular weight complexes by glycerol gradient analysis (Figure 5E) and out of the high-saltextractable nuclear fraction (Supplemental Figure 7D). To determine whether IGF2BP3 influences 7SK snRNA stability, neonatal progenitors underwent lentiviral shRNA knockdown with puromycin selection and megakaryocytic differentiation of transduced cells. This approach yielded isoform-selective repression of IGF2BP3 (Supplemental Figure 7E) and consistently diminished its levels by approximately $80 \%$ (see summary graph in Figure 6E). Notably, the knockdown of IGF2BP3 caused significant 7SK downregulation during neonatal megakaryopoiesis (Figure 5F). These results support the notion that IGF2BP3 participates in fetal/neonatal 7SK snRNP complexes and may thereby enhance 7SK stability in neonatal megakaryocytes.

IGF2BP3 contributes to the phenotypic and molecular features of neonatal megakaryocytes. As isoform-selective knockdown of IGF2BP3 promoted 7SK downregulation in neonatal megakaryocytes, additional studies examined the phenotypic consequences of this manipulation. As anticipated, decreasing the levels of IGF2BP3 conferred several adult-type features, such as enhanced cellular enlargement, growth arrest, polyploidization, and eryth- 

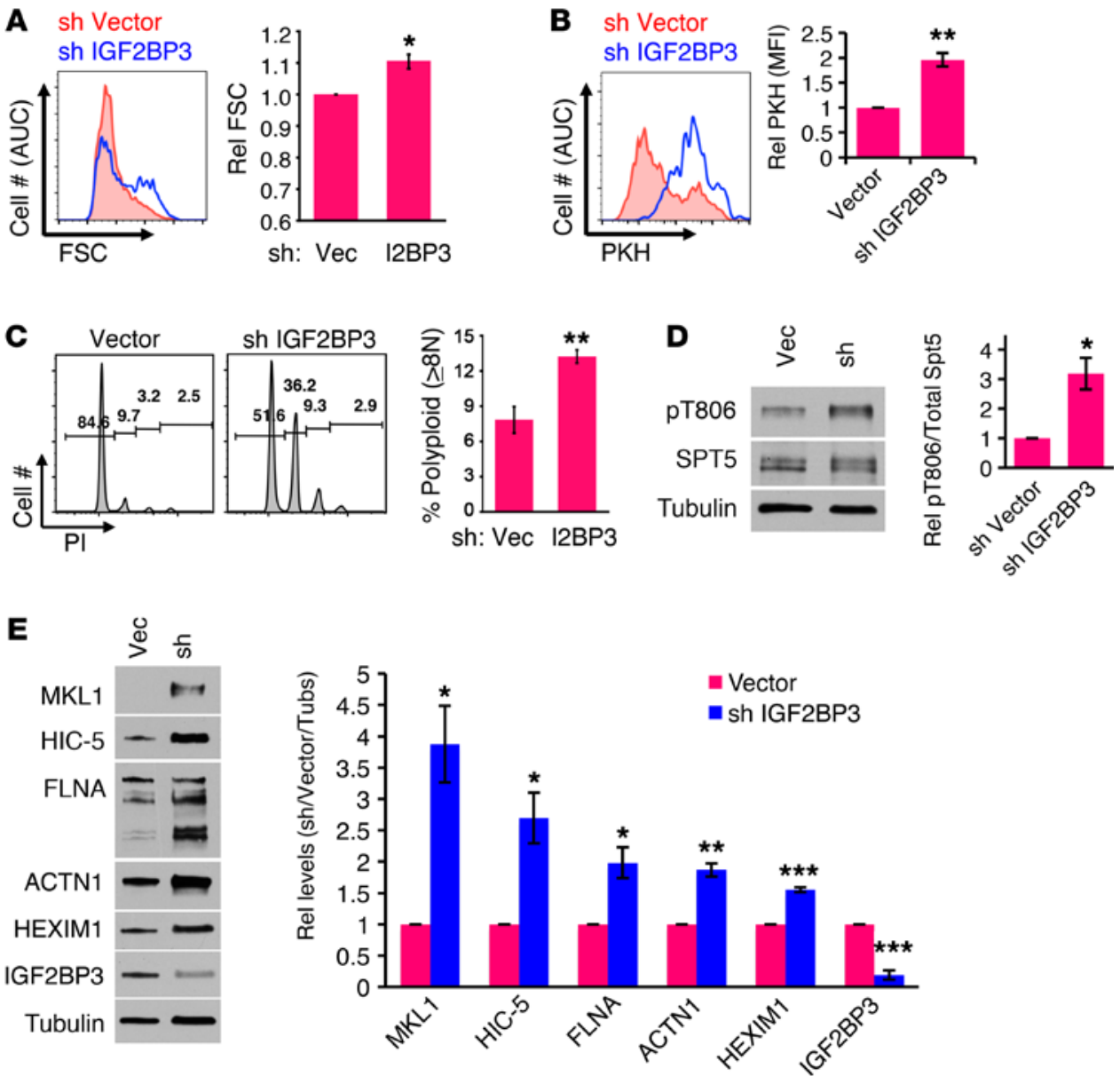

roid suppression (Figure 6, A-C, and Supplemental Figure 8, A and B). At the molecular level, this knockdown enhanced P-TEFb activation, as reflected by SPT5 phosphorylation as well as levels of HEXIM1, RNAPIIO, and H2Bub1 (Figure 6, D and E, and Supplemental Figure 8C). This activation was further associated with upregulation of the P-TEFb-dependent cytoskeletal remodeling factors, particularly the morphogenetic regulator MKL1 (Figure $6 \mathrm{E})$. As a specificity control, adult megakaryocytes transduced with the same lentiviral shRNA constructs underwent comprehensive phenotypic and molecular characterization. In these cells, the IGF2BP3-targeting shRNA caused no significant alterations in any parameters (Supplemental Figure 9), confirming a neonatalspecific effect. These loss-of-function studies thus reveal an influence of IGF2BP3 levels on P-TEFb activation and megakaryocytic morphogenesis, consistent with a role as a critical ontogenic determinant. To address effects of IGF2BP3 knockdown on platelet production, a recently described ex vivo platelet-release assay $(38,39)$ was applied to the culture system. Interpretation was complicated by the accelerated growth arrest and maturation associated with loss of IGF2BP3, but the studies supported an association between knockdown and enhanced platelet production on a per megakaryocyte basis (Supplemental Figure 10).

$B E T$ factor regulation of IGF2BP3 enables pharmacologic modulation of ontogenic influence. Recently published data demonstrate induction of IGF2BP3 by a cancer stem cell pluripotency program (40). In addition, a factor or factors in the BET family have been shown to participate in pluripotency reprogramming of somatic cells (41), suggesting potential BET regulation of IGF2BP3. For these reasons, effects of BET inhibition on neonatal megakaryopoiesis were tested. As shown in Figure 7, 2 different inhibitors, JQ1 and I-BET151, both downregulated the erythroid marker GPA while enhancing expression of megakaryocytic markers CD41 and CD42 (Figure 7, A and B, and Supplemental Figure 11A). These treatments also promoted platelet release, cellular enlargement, and growth arrest (Figure 7, C and D, and Supplemental Figure 11B). At the molecular level, BET inhibition suppressed IGF2GP3 expression while inducing RNAPII hyperphosporylation, upregulation of cytoskeletal remodeling factors, and HEXIM1 upregulation (Figure 8, A and B). MKL1 and FLNA levels showed particularly robust responsiveness to $\mathrm{BET}$ inhibition. In control experiments, treatment of adult progenitors with BET inhibitors failed to upregulate the cytoskeletal remodeling factors or HEXIM1, failed to induce RNAPII hyperphosphorylation, and did not induce cellular enlargement (Supplemental Figure 11, C-E). During the preparation of this manuscript, Palanichamy et al. also showed repression of IGF2BP3 expression by BET inhibition (36).

To further ascertain that the effects of BET inhibition on neonatal megakaryopoiesis resulted from repressing IGF2BP3 expression, retroviral transduction was used to enforce IGF2BP3 expression in neonatal progenitors. Enforced IGF2BP3 significantly blunted the capacity of BET inhibitors to induce enlargement of neonatal megakaryocytes (Figure 9A). Exogenous IGF2BP3 expression basally 
A

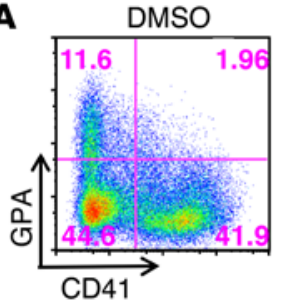

B

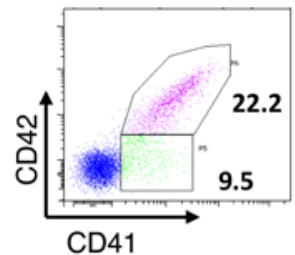

C

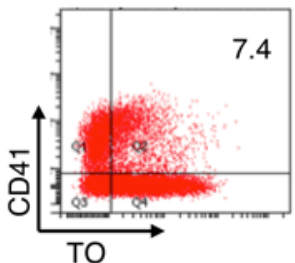

D

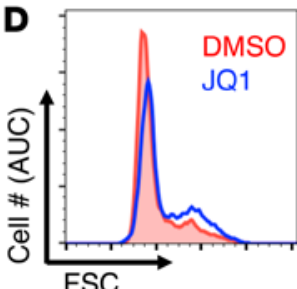

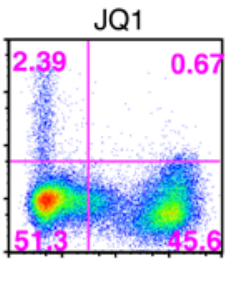

JQ1

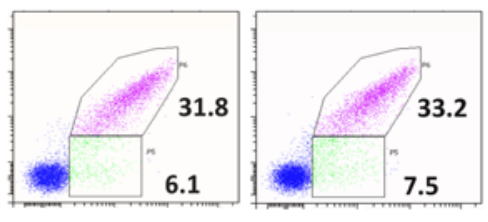

7.5

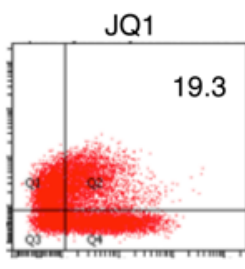

19.3
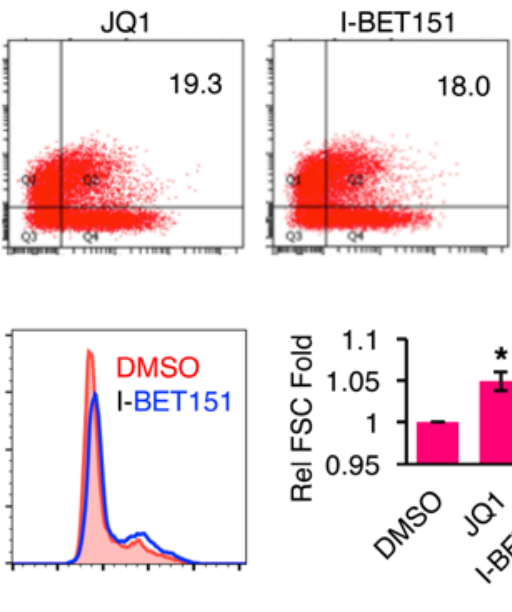

I-BET151

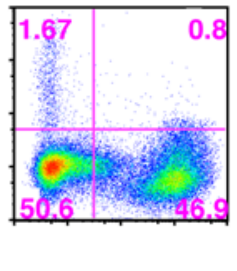

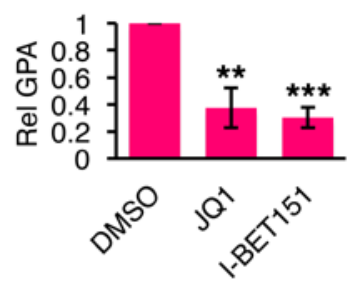
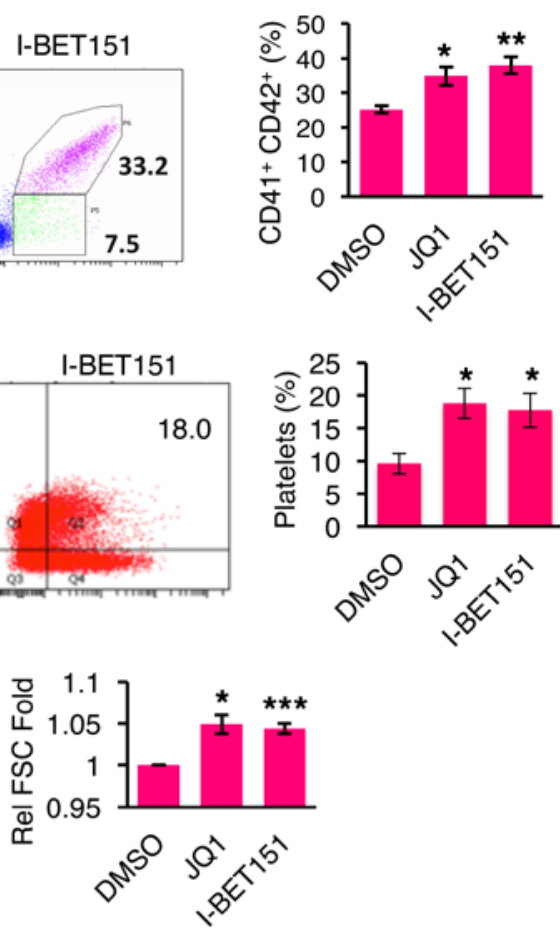

Figure 7. BET factor inhibition in neonatal megakaryocytes enhances multiple features of differentiation. (A) BET factor inhibition in neonatal megakaryocytes promotes lineage consolidation. CB progenitors cultured 6 days in megakaryocyte medium with either control solvent, $0.25 \mu \mathrm{M}$ JQ1, or $0.5 \mu \mathrm{M}$ I-BET151 were analyzed by flow cytometry for erythroid (GPA) and megakaryocytic (CD41) markers, with gating on viable cells. Graph represents mean percentages of GPA relative to solvent control \pm SEM for 3 independent experiments. ${ }^{* *} P<0.01$; ${ }^{* *} P<0.005, t$ test. (B) Enhancement of CD42 expression in neonatal megakaryocytes treated with BET inhibitors. Neonatal progenitors treated with solvent or BET inhibitors were cultured for 7 days as in $\mathbf{A}$ and analyzed for CD41 and CD42 expression, with gating on viable cells. Graph shows mean percentages of CD41+CD42 ${ }^{+}$double-positive cells \pm SEM for 4 independent experiments. ${ }^{*} P<0.05$; ${ }^{* *} P<0.01, t$ test. (C) Enhancement of neonatal platelet production by BET inhibition. Neonatal megakaryocyte cultures were treated with solvent, $0.125 \mu \mathrm{M}$ JQ1, or $0.25 \mu \mathrm{M}$ I-BET151 for 5 days. Inhibitors were then withdrawn, and the cultures underwent megakaryocyte maturation for an additional 6 days. Culture-derived platelets were collected from the supernatant and analyzed by flow cytometry following labeling with antiCD41 antibodies and thiazole orange (TO). An analytical gate for size and CD41/TO staining was established on fresh PB platelets. Graph represents mean platelet percentages \pm SEM for 3 independent experiments. ${ }^{*} P<0.05, t$ test. (D) BET factor inhibition in neonatal megakaryocytes enhances enlargement. Neonatal progenitors cultured as in A were analyzed for size (FSC) within the viable CD41+ gated population. Graph represents mean \pm SEM for 3 independent experiments. ${ }^{*} P<0.05 ;{ }^{* *} P<0.005, t$ test. See also Supplemental Figure 11.

upregulated GPA and rendered both GPA and CD41 less responsive to BET inhibition (Figure 9, B and C). These findings confirm that some of the phenotypic effects of BET inhibitors on neonatal megakaryocytes result from downregulation of IGF2BP3.

\section{Discussion}

It has long been appreciated that the ontogenic stage exerts a profound influence on human megakaryocyte morphogenesis. In addition to the clinical implications for neonatal infants and CB HSC transplant recipients, this influence affects the feasibility of ex vivo, donor-independent platelet production. Due to their capacity for unlimited expansion and genetic manipulation, PSCs have become a leading candidate for ex vivo megakaryocyte generation. However, PSC-derived megakaryocytes display poor platelet yields (42), and extensive profiling has shown these cells to be even more ontogenically primitive than human FL megakaryocytes, likely reflecting a yolk-sac stage (10). Thus, ontogenic manipulation has the potential to improve both yield and quality of ex vivo-derived platelets.

While a megakaryocyte ontogenic regulatory mechanism has not previously been identified, contributory factors have been described. Klusmann et al. identified fetal-specific hyperactivation of the IGF/AKT/mTOR pathway, which primed MkPs for leukemic transformation (43). These findings were supported by a subsequent study in which the proliferative phenotype of neonatal megakaryocytes was linked to mTOR hyperactivation (8). Impor- 
A

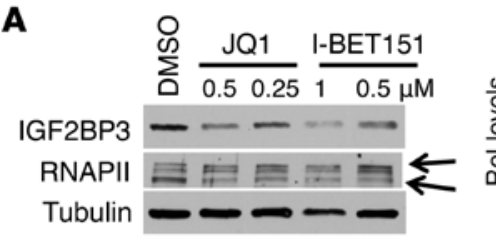

B

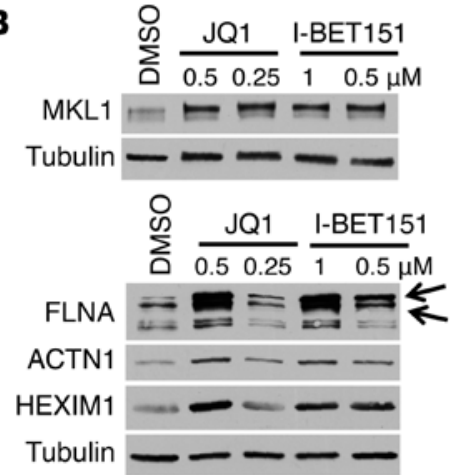

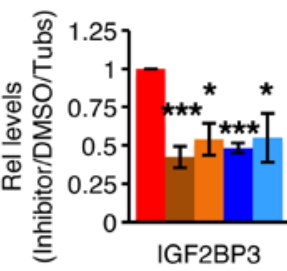

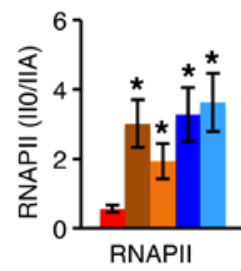

- DMSO

घJQ1 $0.5 \mu \mathrm{M}$

= JQ $10.25 \mu \mathrm{M}$

- I-BET151 $1 \mu \mathrm{M}$

= I-BET151 $0.5 \mu \mathrm{M}$
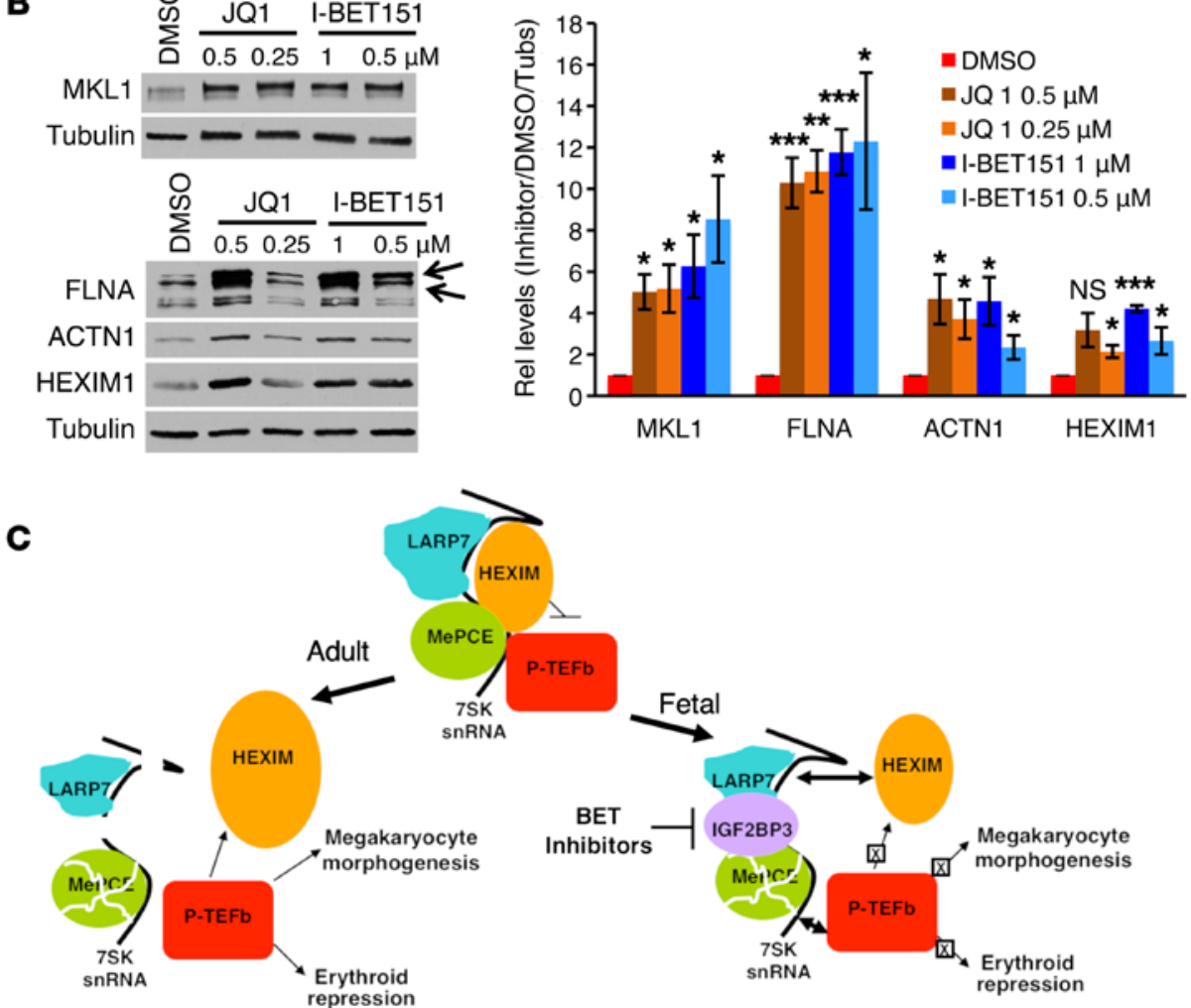

Figure 8. BET factor inhibition in neonatal megakaryocytes elicits adult molecular features. (A and B) BET factor inhibition in neonatal megakaryocytes downregulates IGF2BP3 and elicits adult-like molecular features. Whole cell lysates of cells treated with BET inhibitors as in Figure 7A were immunoblotted for indicated factors as in Figure 2. Arrows in A show RNAPIIO and IIA isoforms. Arrows in B show intact FLNA and the approximately 190-kDa cleavage fragment. Graphs show mean \pm SEM for densitometry values from 3 independent experiments, with signal normalization as in Figure $2 A$. ${ }^{*} P<0.05$; ${ }^{*} P<0.01$; ${ }^{* *} P<0.005, t$ test. (C) Model of ontogenic regulation of megakaryocyte morphogenesis. In adult megakaryopoiesis (left arrow), downregulation of LARP7 and proteolysis of MePCE destabilize 7SK snRNA, leading to unopposed P-TEFb activation. This mode of P-TEFb activation promotes upregulation of megakaryocyte morphogenesis factors, most notably MKL1, as well as upregulation of HEXIM1 and lineage consolidation via erythroid repression. In fetal megakaryopoiesis (right arrow), expression of ICF2BP3 stabilizes 7SK snRNA despite downregulation of LARP7 and MePCE. Persistence of 7SK allows for feedback inhibition of P-TEFb, dampening both the upregulation of megakaryocyte morphogenesis factors such as MKL1 and lineage consolidation via erythroid repression. See also Supplemental Figure 11.

tantly, this latter study demonstrated confinement of the neonatal phenotype to morphogenesis and proliferation, with no alterations in other aspects of megakaryocyte differentiation, including expression of platelet-surface glycoproteins (e.g., CD42), granule maturation, transcription factor expression, and TPO receptor function. The known participation of IGF2BP3 in inducing IGF/ AKT/mTOR signaling during oncogenesis (40) suggests that its abundant levels in neonatal megakaryocytes (see Figure 3A) may contribute to mTOR hyperactivation.

Comparisons of murine FL and adult marrow MkPs have also identified a microenvironmental influence, consisting in part of increased type I interferon in the adult milieu (16). Interestingly, murine FL MkPs, when cultured ex vivo, acquire the capacity for adult-type morphogenesis (44), unlike findings with human pro- genitors (10). Importantly, the ex vivo differentiation of murine FL MkPs is associated with reproducible downregulation of $\operatorname{Igf} 2 b p 3$ (see GEO GDS1316 from ref. 44) in contrast with cultured human neonatal progenitors, which show no change in IGF2BP3 during maturation of committed MkPs (see BloodSpot, DMAP data set; ref. 45). Thus there may be differences in the regulation of the ontogenic program in murine versus human hematopoiesis, with murine progenitors possessing more plasticity in responding to milieu influences.

The current results identify IGF2BP3 as an ontogenic master switch that regulates megakaryocyte morphogenesis, at least in part, through modulation of a specialized P-TEFb activation program (see Figure $8 \mathrm{C}$ ). Among the notable targets in this pathway is MKL1, a factor previously implicated as a key regulator of 
A
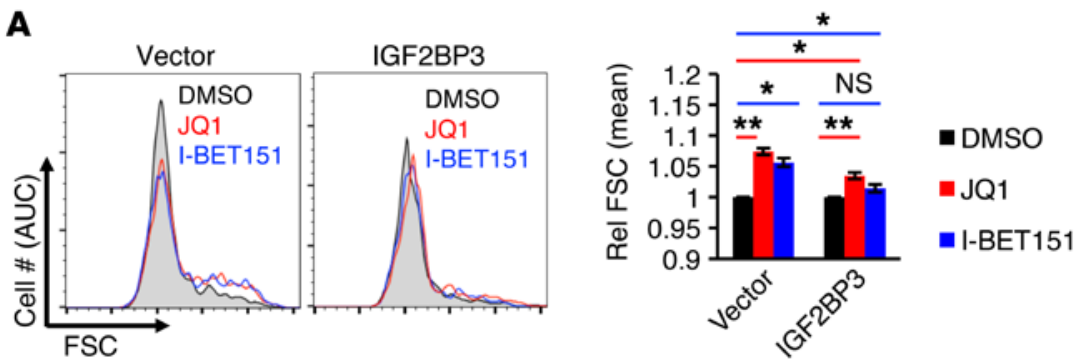

B
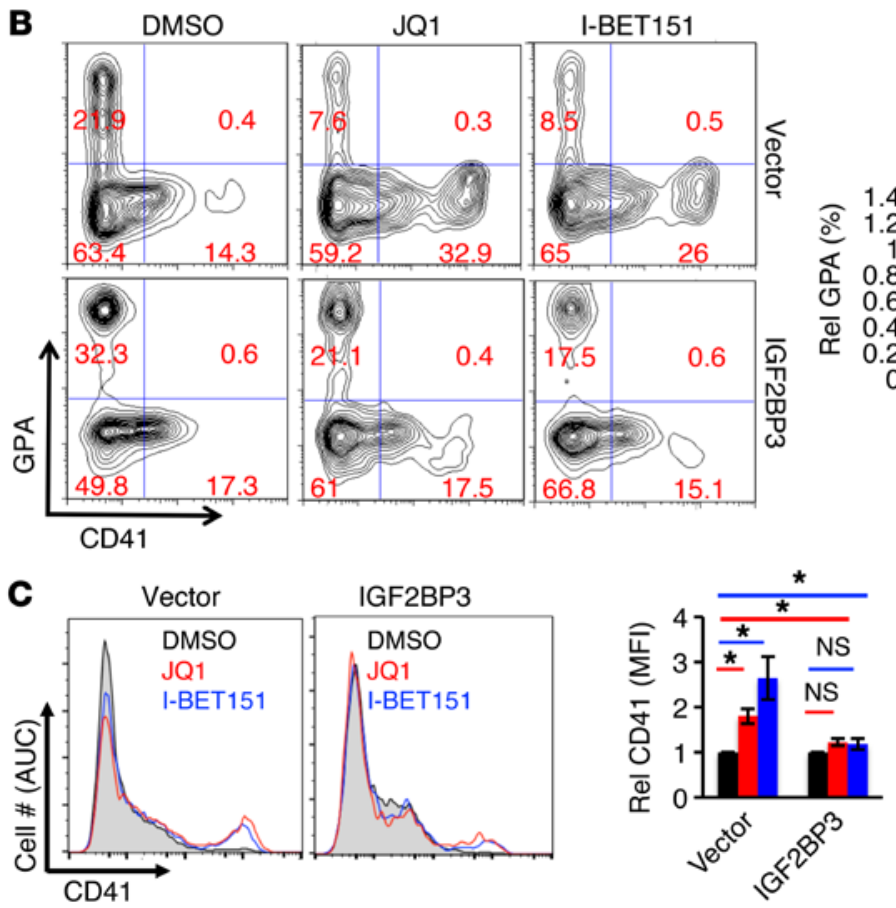

megakaryocyte morphogenesis. MKL1 undergoes upregulation, nuclear translocation, and transcriptional activation during megakaryopoiesis (46). Mkl1-deficient murine megakaryocytes display defects in polyploidization, enlargement, proliferation control, and proplatelet production $(14,47,48)$, thus phenocopying normal human neonatal megakaryocytes. Gene-expression profiles indicate that MKL1-regulated genes constitute a specific, small subset of megakaryocytic genes, encoding cytoskeletal remodeling factors and the ploidy regulator GEF-H1 $(14,15,48)$. Two MKL1-regulated factors, FLNA and HIC-5, bind to MKL1, promote its activation, and thus induce feed-forward loops $(49,50)$. Notably, our results show that human neonatal megakaryocytes have significant deficiencies of MKL1, FLNA, and HIC-5 and augment these factors in response to IGF2BP3 knockdown (see Figure 5E). MKL1 and FLNA also show striking upregulation in neonatal megakaryocytes in response to BET inhibition (Figure 6D). Therefore, MKL1 likely represents a proximal mediator in the ontogenic regulation of morphogenesis by IGF2BP3-P-TEFb.

How changes in the levels of P-TEFb liberation can exert specific effects on the megakaryocytic morphogenesis program remains unknown. In many cell types, developmentally regulated genes undergo activation through a guided recruitment of active $\mathrm{P}$-TEFb. This process is mediated by a series of steps involving chromatin adaptor factors that recruit the 7SK snRNP, P-TEFb
Figure 9. Phenotypic effects of BET factor inhibition occur via IGF2BP3 repression. (A-C) Enforced expression of IGF2BP3 blunts the effects of BET factor inhibition in neonatal megakaryocytes. CB progenitors retrovirally transduced with control or IGF2BP3 expression vectors underwent 6 days of culture in megakaryocyte medium $\pm \mathrm{BET}$ inhibitors as in Figure 7A. Cells were analyzed by flow cytometry for size (FSC) within the viable $\mathrm{GFP}^{+} \mathrm{CD} 41^{+}$gated population (A). In addition, viable GFP+ ${ }^{+}$cells were analyzed for expression of the erythroid marker GPA and the megakaryocytic marker CD41 (B and C). Graphs in A-C represent mean \pm SEM for 4 independent experiments. Analysis between the 2 groups employed 2-way ANOVA. Asterisks above the long line indicate row factor $x$ column factor interactions. ${ }^{*} P<0.05 ;{ }^{* *} P<0.01$; ${ }^{* * *} P<0.005$. release factors that locally liberate CDK9/cyclin T, and the super elongation complex (SEC), which delivers the kinase to a recipient complex including RNAPII, polymerase-associated factor (PAF), and DSIF (51). Compared with other hematopoietic lineages, megakaryocytes appear to be deficient in some components of the SEC, PAF, and DSIF complexes (ref. 45 and unpublished data, A.N. Goldfarb laboratory). They may therefore require higher thresholds and alternative pathways of P-TEFb release to insure sufficient delivery to those genes most susceptible to RNAPII pausing. Thus lineage- and gene-specific mechanisms most likely converge to translate specific thresholds of free P-TEFb into a coordinated transcriptional output.

The upregulation of P-TEFb-dependent factors by BET inhibition appears paradoxical, given the role of bromodomain-containing 4 (BRD4) in the recruitment of P-TEFb to target genes. However, 2 mechanisms may contribute to this phenomenon. First, the threshold for IGF2BP3 repression by BET inhibitors may fall below the threshold for repression of megakaryocytic $\mathrm{P}$-TEFb target genes, thus providing a window for indirect activation. Second, BRD4 may not be required or may play a negative role in the activation of some megakaryocytic P-TEFb target genes, as has been described for HIV transcription (52). The results shown in Figure 9 indicate that several of the promegakaryocytic effects of BET inhibition occur through IGF2BP3 
repression. However, blockade of BET factor recruitment to acetylated chromatin also disrupts mediator recruitment to super enhancers, repressing hundreds of associated genes (53). Thus, complicated network perturbations and off-target effects are expected with this particular intervention.

The current results elucidate a transcriptional mechanism underlying the ontogenic regulation of megakaryopoiesis. Because IGF2BP3 is broadly expressed during embryogenesis (54), its stabilizing influence on the 7SK snRNP could potentially alter P-TEFb regulation in a variety of developing fetal tissues and provide a general principle for ontogenic regulation. However, its effects are expected to be most prominently manifested in cells that normally downregulate the 7SK-stabilizing factors LARP7 and MePCE, e.g., megakaryocytes. The role of this regulatory pathway does not exclude additional mechanisms for IGF2BP3 in ontogenic regulation, nor does it exclude contributions from unrelated pathways. Our results do show though that manipulation of this pathway offers a promising strategy for enhancing megakaryocyte platelet production capacity.

\section{Methods}

Cell culture. Purified human adult $\mathrm{CD} 34^{+} \mathrm{PB}$ cells were purchased from the Fred Hutchinson Cancer Research Center (Seattle, Washington, USA), and purified human umbilical CD $34^{+} \mathrm{CB}$ cells were purchased from AllCells. The cells were cultured in serum-free unilineage media as described previously (11). Briefly, undifferentiated cells were expanded for 72 hours in prestimulation medium containing Iscove modified Dulbecco medium with 20\% BITS 9500 (StemCell Technologies), $2 \mathrm{mM}$ L-glutamine, $100 \mathrm{ng} / \mathrm{ml}$ stem cell factor (SCF), $100 \mathrm{ng} / \mathrm{ml} \mathrm{FLT3-ligand} \mathrm{(FLT3L),} 100 \mathrm{ng} / \mathrm{ml} \mathrm{TPO}$, and $20 \mathrm{ng} / \mathrm{ml} \mathrm{IL}-3$. Erythroid and megakaryocytic cells were cultured in their respective media for the indicated durations following prestimulation. Erythroid cultures contained the same medium as for prestimulation, but with cytokines consisting of $4.5 \mathrm{U} / \mathrm{ml}$ human erythropoietin (Epogen, Amgen) and $25 \mathrm{ng} / \mathrm{ml} \mathrm{SCF}$. Megakaryocyte cultures also contained the same medium, but with $40 \mathrm{ng} / \mathrm{ml}$ TPO, $25 \mathrm{ng} / \mathrm{ml} \mathrm{SCF}, 100 \mathrm{ng} / \mathrm{ml}$ stromal-derived factor- $\alpha$, and used fibronectin-coated wells. Cytokines were purchased from PeproTech. JQ1 and I-BET151 (Sigma-Aldrich) dissolved in DMSO were added to cultures as indicated. K562 and HEK293T cells underwent standard culture $(11,12)$.

Plasmids, transfection, and transduction. The human IGF2BP3 expression construct pDESTmycIGF2BP3, which expresses an N-terminal Myc epitope-tagged protein using a CMV promoter, was obtained from Addgene (plasmid 19879) (55) and transfected into HEK293T cells as described (11). For subcloning into the MIG retroviral expression vector, full-length coding sequence was isolated by high-fidelity PCR. The retroviral expression construct for human Lin28b was provided by Stefan Muljo (National Institute of Allergy and Infectious Diseases, Bethesda, Maryland, USA). For human progenitor transduction, retroviral packaging and cellular transduction by spinoculation were conducted as described (56). For murine progenitor transduction, MIG vector and MIG-IGF2BP3 were packaged by 293 T cotransfection with pCL-Eco (Addgene) using the CalPhos kit (Clontech). Marrow from 5-fluorouracil-treated C57BL/6J mice (CD45.2, Jackson Laboratories) underwent prestimulation and spinoculation. See Supplemental Methods for further details.
Lentiviral expression constructs for HMGA1a, HMGA1b, and parent vector (pLent-GFP-HMGI, pLent-GFP-HMGY, and pLentGFP) were provided by Linda Resar (Johns Hopkins School of Medicine, Baltimore, Maryland, USA). For lentiviral shRNA-mediated knockdown, pLKO.1-derived constructs were purchased from Open Biosystems GE-Dharmacon. Lentiviral packaging by cotransfection of HEK293T cells was conducted as described (11), except that experiments after 2014 used the hCMV-BaevRless envelope construct (57). This construct uses the CMV promoter to express during packaging the baboon retroviral envelope glycoprotein, which provides superior transduction efficiency for human $\mathrm{CD} 34^{+}$progenitors. For shRNA knockdowns, transduced CB CD34 cells were selected with puromycin at $2 \mu \mathrm{g} / \mathrm{ml}$ during an initial 72-hour expansion phase followed by 1 $\mu \mathrm{g} / \mathrm{ml}$ during the differentiation phase.

Bone marrow transplant. Eight-week-old female B6.SJL-Ptprc ${ }^{a}$ Pepc $c^{b}$ IboyJ mice (CD45.1, Jackson Laboratories) were irradiated with 2 doses of 6 Gy separated by 3 hours. Two hours after irradiation, each animal received by tail vein injection $2 \times 10^{6}$ marrow cells from the spinoculation cultures. At 6 weeks after transplant, half of the recipients $(n=12)$ underwent treatment with murine TPO (Peprotech) at $2 \mathrm{mg} / \mathrm{d}$ by i.p. injection for 3 consecutive days followed by a 4-day recovery period. See Supplemental Methods for further details.

Flow cytometry. Expression of CD41 and GPA, ploidy analysis, and $\mathrm{PKH} 26$ dye dilution assays in human progenitors were performed as described previously $(11,12)$. For the human progenitor retroviral transduction experiments enforcing IGF2BP3 expression, analyses were conducted on viable $\mathrm{GFP}^{+}$gated cells. Assessment of platelet release was conducted as previously described $(38,39)$. Studies on transplanted mice were conducted 6 weeks after transplant. Marrow and spleens were disaggregated by passing through $70-\mu \mathrm{m}$ mesh and subjected to lysis of red cells using hypotonic ammonium chloride (Gibco ACK lysis buffer, Thermo Fisher Scientific). Samples were costained with APC-conjugated rat anti-CD41 and PE-conjugated rat anti-TER119 or isotype controls (BD Biosciences) and analyzed on a $\mathrm{BD}$ LSRFortessa with initial gating on viable $\mathrm{GFP}^{+}$cells.

IP and immunoblot. IP of HEXIM1 from K562 cellular extracts followed by immunoblot (IB) analysis was performed as described (11, 12). The RNA-binding protein IP assay (RIP) was performed according to the protocol described by Jain et al. (58) with some modifications. Briefly, HEK293T cells transiently transfected with pDESTmycIGF2BP3 were incubated 5 minutes in ice-cold polysome lysis buffer (10 mM Hepes, pH 7.0, $100 \mathrm{mM} \mathrm{KCl,} 5 \mathrm{mM} \mathrm{MgCl}_{2}, 0.5 \%$ Nonidet P-40 [NP-40], 1 mM DTT) supplemented with EDTA-free protease inhibitor cocktail (Roche), $20 \mu \mathrm{M}$ calpain inhibitor III, and $40 \mathrm{U} / \mathrm{ml}$ RNase inhibitor (Promega) and then stored at $-80^{\circ} \mathrm{C}$. Rapidly thawed extracts were cleared by centrifugation at $16,000 \mathrm{~g}$ at $4^{\circ} \mathrm{C}$ for 10 minutes followed by dilution with NET-2 buffer (50 mM Tris-HCl, pH 7.4, 150 $\mathrm{mM} \mathrm{NaCl}, 1 \mathrm{mM} \mathrm{MgCl}$, 0.05\% NP-40, 20 mM EDTA, pH 8.0, 1 mM DTT) supplemented with protease and RNase inhibitors as above. For IP, diluted extracts were incubated overnight at $4^{\circ} \mathrm{C}$ with anti-Myc-tag or isotope-matched control antibody, followed by capture with protein A/G magnetic beads and multiple washes with buffer NT-2 (50 mM Tris-HCl, pH 7.4, $\left.150 \mathrm{mM} \mathrm{NaCl}, 1 \mathrm{mM} \mathrm{MgCl}_{2}, 0.05 \% \mathrm{NP}-40\right)$. One portion of the beads was boiled in $1 \times$ SDS-PAGE loading buffer for IB assessment of IP efficiency. The other portion was processed for RNA analysis by treatment first with DNAse, then addition of $10 \%$ Chelex-100 in water, which was boiled for 10 minutes, followed by addi- 
tion of proteinase $\mathrm{K}$ with digestion at $55^{\circ} \mathrm{C}$ for 30 minutes. Direct IB analysis of sheared whole cell lysates was conducted as described (12). Densitometry data were acquired on a GS800 calibrated densitometer (Bio-Rad) and analyzed with Quantity One software (Bio-Rad).

RNA quantitation. For quantitation of 7SK snRNA, total cellular RNA isolated using the RNeasy Plus Mini Kit (QIAGEN) or RNA from RIP was converted to cDNA using the iscript kit (Bio-Rad) and analyzed by quantitative PCR (qPCR) on the iCycler platform using iQ SYBR Green Supermix (Bio-Rad). Primers and cycling conditions were described previously (11). Relative transcript levels were calculated using GAPDH normalization with the $\triangle \triangle \mathrm{Ct}$ formula.

Glycerol gradient analysis. Transfected HEK293T cells were harvested and resuspended in ice-cold extraction buffer (10 mM HEPES, $150 \mathrm{mM} \mathrm{NaCl}, 2 \mathrm{mM} \mathrm{MgCl}$, 0.5\% NP-40, 1 mM DTT, 1 mM PMSF, 20 $\mu \mathrm{M}$ calpain inhibitor III, EDTA-free protease inhibitor cocktail, $40 \mathrm{U} /$ $\mathrm{ml}$ RNase inhibitor). Extracts were loaded on $10 \%-45 \%$ glycerol gradients prepared in extraction buffer. The gradients were spun on an SW-41 rotor at $40,000 \mathrm{~g}$ for 16 hours at $4^{\circ} \mathrm{C}$, and resultant fractions underwent SDS-PAGE IB.

Statistics. Excel, version 14.4 (Microsoft), KaleidaGraph software, version 4.5 (Synergy Software), or GraphPad Prism Mac 6.0f (GraphPad Software) was used to display the data graphically and to perform statistical analyses. Results were analyzed by unpaired 2-tailed Student's $t$ test. Multiple intergroup comparisons were conducted using 2-way repeated measures ANOVA. $P$ values less than or equal to 0.05 were considered significant.

Study approval. The normal donor samples purchased from the Fred Hutchinson Cancer Research Center and AllCells were originally obtained with informed consent and IRB approval at both institutions. Analysis of preexisting human marrow samples obtained for prior clin- ical indications was carried out in accordance with IRB approval from the participating institutions, University of Virginia (HSR\#13310) and University of Arizona (0500000226), following the principles of the Declaration of Helsinki. Animal studies were approved by the University of Virginia IACUC (protocol 3545).

\section{Author contributions}

KEE designed, conducted, and interpreted experiments and wrote the manuscript. CHL, SK, GM, EZ, and PB conducted experiments. DRF and CIR contributed to experimental design, oversight, and interpretation. AAG identified marrow samples and scored immunohistochemistry. DAF and LMR identified and provided marrow samples. EV, MS, and RPF provided critical, unique reagents and assisted with writing the manuscript. ANG designed and interpreted experiments and wrote the manuscript.

\section{Acknowledgments}

We thank Stefan Muljo and Linda Resar for plasmids, Ramesh Yadava for generous assistance with in situ hybridization, Pat Pramoonjago for immunohistochemistry, and Tim Bender for valuable guidance with murine transplantation experiments. This work was supported by NIH grants DK090926 and HL130550. PB was supported in part by grant NIH T32 CA009109-39 (Cancer Research Training in Molecular Biology) awarded to the University of Virginia.

Address correspondence to: Adam Goldfarb or Kamal Elagib, Department of Pathology, University of Virginia School of Medicine, PO Box 800904, Charlottesville, Virginia 22908, USA. Phone: 434.982.0593; E-mail: ang3x@virginia.edu (A. Goldfarb). Phone: 434.982.0430; E-mail: ka8b@virginia.edu (K. Elagib).
1. Allen Graeve JL, de Alarcon PA. Megakaryocytopoiesis in the human fetus. Arch Dis Child. 1989;64(4 Spec No):481-484.

2. de Alarcon PA, Graeve JL. Analysis of megakaryocyte ploidy in fetal bone marrow biopsies using a new adaptation of the feulgen technique to measure DNA content and estimate megakaryocyte ploidy from biopsy specimens. Pediatr Res. 1996;39(1):166-170.

3. Fuchs DA, McGinn SG, Cantu CL, Klein RR, Sola-Visner MC, Rimsza LM. Developmental differences in megakaryocyte size in infants and children. Am J Clin Pathol. 2012;138(1):140-145.

4. Rajasekhar D, Kestin AS, Bednarek FJ, Ellis PA, Barnard MR, Michelson AD. Neonatal platelets are less reactive than adult platelets to physiological agonists in whole blood. Thromb Haemost. 1994;72(6):957-963.

5. Bednarek FJ, Bean S, Barnard MR, Frelinger $\mathrm{AL}$, Michelson AD. The platelet hyporeactivity of extremely low birth weight neonates is agedependent. Thromb Res. 2009;124(1):42-45.

6. Ferrer-Marin F, Stanworth S, Josephson C, Sola-Visner M. Distinct differences in platelet production and function between neonates and adults: implications for platelet transfusion practice. Transfusion. 2013;53(11):2814-2821; quiz 2813.

7. Ignatz $\mathrm{M}$, et al. Umbilical cord blood produces small megakaryocytes after transplantation. Biol Blood Marrow Transplant. 2007;13(2):145-150.
8. Liu ZJ, et al. Developmental differences in megakaryocytopoiesis are associated with up-regulated TPO signaling through mTOR and elevated GATA-1 levels in neonatal megakaryocytes. Blood. 2011;117(15):4106-4117.

9. Mattia G, et al. Different ploidy levels of megakaryocytes generated from peripheral or cord blood CD $34^{+}$cells are correlated with different levels of platelet release. Blood. 2002;99(3):888-897.

10. Bluteau O, et al. Developmental changes in human megakaryopoiesis. J Thromb Haemost. 2013;11(9):1730-1741.

11. Elagib KE, et al. Calpain 2 activation of $\mathrm{P}-\mathrm{TEF}$ drives megakaryocyte morphogenesis and is disrupted by leukemogenic GATA1 mutation. Dev Cell. 2013;27(6):607-620.

12. Elagib KE, et al. Cross-talk of GATA-1 and $\mathrm{P}-\mathrm{TEFb}$ in megakaryocyte differentiation. Blood. 2008;112(13):4884-4894.

13. Zhou Q, Li T, Price DH. RNA polymerase II elongation control. Annu Rev Biochem. 2012;81:119-143.

14. Smith EC, et al. MKL1 and MKL2 play redundant and crucial roles in megakaryocyte maturation and platelet formation. Blood. 2012;120(11):2317-2329.

15. Gao Y, et al. Role of RhoA-specific guanine exchange factors in regulation of endomitosis in megakaryocytes. Dev Cell. 2012;22(3):573-584.
16. Woo AJ, et al. Developmental differences in IFN signaling affect GATA1s-induced megakaryocyte hyperproliferation. J Clin Invest. 2013;123(8):3292-3304.

17. Chou ST, et al. Trisomy 21-associated defects in human primitive hematopoiesis revealed through induced pluripotent stem cells. Proc Natl Acad Sci U S A. 2012;109(43):17573-17578.

18. Sansó M, et al. P-TEFb regulation of transcription termination factor Xrn 2 revealed by a chemical genetic screen for Cdk9 substrates. Genes Dev. 2016;30(1):117-131.

19. Sano M, et al. Activation and function of cyclin T-Cdk9 (positive transcription elongation factor-b) in cardiac muscle-cell hypertrophy. Nat Med. 2002;8(11):1310-1317.

20. Hsin JP, Sheth A, Manley JL. RNAP II CTD phosphorylated on threonine- 4 is required for histone mRNA 3' end processing. Science. 2011;334(6056):683-686.

21. Yu M, et al. RNA polymerase II-associated factor 1 regulates the release and phosphorylation of paused RNA polymerase II. Science. 2015;350(6266):1383-1386.

22. Bartkowiak B, et al. CDK12 is a transcription elongation-associated CTD kinase, the metazoan ortholog of yeast Ctk1. Genes Dev. 2010;24(20):2303-2316.

23. Albert TK, Antrecht C, Kremmer E, Meisterernst M. The establishment of a hyperactive structure 
allows the tumour suppressor protein $\mathrm{p} 53$ to function through P-TEFb during limited CDK9 kinase inhibition. PLoS One. 2016;11(1):e0146648.

24. Pirngruber J, et al. CDK9 directs H2B monoubiquitination and controls replication-dependent histone mRNA 3'-end processing. EMBO Rep. 2009;10(8):894-900.

25. Shchebet A, Karpiuk O, Kremmer E, Eick D, Johnsen SA. Phosphorylation by cyclin-dependent kinase-9 controls ubiquitin-conjugating enzyme-2A function. Cell Cycle. 2012;11(11):2122-2127.

26. Alazami AM, et al. Loss of function mutation in LARP7, chaperone of 7SK ncRNA, causes a syndrome of facial dysmorphism, intellectual disability, and primordial dwarfism. Hum Mutat. 2012;33(10):1429-1434.

27. Barboric M, Lenasi T, Chen H, Johansen EB, Guo $\mathrm{S}$, Peterlin BM. 7SK snRNP/P-TEFb couples transcription elongation with alternative splicing and is essential for vertebrate development. Proc Natl Acad Sci U S A. 2009;106(19):7798-7803.

28. Xue Y, Yang Z, Chen R, Zhou Q. A capping-independent function of MePCE in stabilizing 7SK snRNA and facilitating the assembly of 7SK snRNP. Nucleic Acids Res. 2010;38(2):360-369.

29. Krueger BJ, et al. LARP7 is a stable component of the 7SK snRNP while P-TEFb, HEXIM1 and hnRNP A1 are reversibly associated. Nucleic Acids Res. 2008;36(7):2219-2229.

30. Dai Q, et al. Primordial dwarfism gene maintains Lin28 expression to safeguard embryonic stem cells from premature differentiation. Cell Rep. 2014;7(3):735-746

31. Eckfeldt $\mathrm{CE}$, et al. Functional analysis of human hematopoietic stem cell gene expression using zebrafish. PLoS Biol. 2005;3(8):e254.

32. Bell JL, et al. Insulin-like growth factor 2 mRNA-binding proteins (IGF2BPs): post-transcriptional drivers of cancer progression? Cell Mol Life Sci. 2013;70(15):2657-2675.

33. Jeronimo C, et al. Systematic analysis of the protein interaction network for the human transcription machinery reveals the identity of the 7SK capping enzyme. Mol Cell. 2007;27(2):262-274.

34. Copley MR, et al. The Lin28b-let-7-Hmga2 axis determines the higher self-renewal potential of fetal haematopoietic stem cells. Nat Cell Biol.
2013;15(8):916-925.

35. Rivera Vargas T, et al. Post-transcriptional regulation of cyclins D1, D3 and G1 and proliferation of human cancer cells depend on IMP-3 nuclear localization. Oncogene. 2014;33(22):2866-2875.

36. Palanichamy JK, et al. RNA-binding protein IGF2BP3 targeting of oncogenic transcripts promotes hematopoietic progenitor proliferation. J Clin Invest. 2016;126(4):1495-1511.

37. Uchikawa E, et al. Structural insight into the mechanism of stabilization of the 7SK small nuclear RNA by LARP7. Nucleic Acids Res. 2015;43(6):3373-3388.

38. Iancu-Rubin C, et al. Down-regulation of stathmin expression is required for megakaryocyte maturation and platelet production. Blood. 2011;117(17):4580-4589.

39. Iancu-Rubin C, Mosoyan G, Glenn K, Gordon RE, Nichols GL, Hoffman R. Activation of p53 by the MDM2 inhibitor RG7112 impairs thrombopoiesis. Exp Hematol. 2014;42(2):137-145.e5.

40. Chen CL, et al. Reciprocal regulation by TLR4 and TGF- $\beta$ in tumor-initiating stem-like cells. J Clin Invest. 2013;123(7):2832-2849.

41. Liu L, et al. Transcriptional pause release is a rate-limiting step for somatic cell reprogramming. Cell Stem Cell. 2014;15(5):574-588.

42. Sim X, Poncz M, Gadue P, French DL. Understanding platelet generation from megakaryocytes: implications for in vitro-derived platelets. Blood. 2016;127(10):1227-1233.

43. Klusmann JH, et al. Developmental stage-specific interplay of GATA1 and IGF signaling in fetal megakaryopoiesis and leukemogenesis. Genes Dev. 2010;24(15):1659-1672.

44. Li Z, Godinho FJ, Klusmann JH, Garriga-Canut M, Yu C, Orkin SH. Developmental stageselective effect of somatically mutated leukemogenic transcription factor GATA1. Nat Genet. 2005;37(6):613-619.

45. Bagger FO, et al. BloodSpot: a database of gene expression profiles and transcriptional programs for healthy and malignant haematopoiesis. Nucleic Acids Res. 2016;44(D1):D917-D924.

46. Smith EC, et al. Induction of megakaryocyte differentiation drives nuclear accumulation and transcriptional function of MKL1 via actin polymerization and RhoA activation. Blood.
2013;121(7):1094-1101.

47. Cheng EC, et al. Role for MKL1 in megakaryocytic maturation. Blood. 2009;113(12):2826-2834.

48. Ragu C, et al. The serum response factor (SRF)/ megakaryocytic acute leukemia (MAL) network participates in megakaryocyte development. Leukemia. 2010;24(6):1227-1230.

49. Kircher P, et al. Filamin A interacts with the coactivator MKL1 to promote the activity of the transcription factor SRF and cell migration. $\mathrm{Sci}$ Signal. 2015;8(402):ra112.

50. Varney SD, et al. Hic- 5 is required for myofibroblast differentiation by regulating mechanically dependent MRTF-A nuclear accumulation. J Cell Sci. 2016;129(4):774-787.

51. C Quaresma AJ, Bugai A, Barboric M. Cracking the control of RNA polymerase II elongation by 7SK snRNP and P-TEFb. Nucleic Acids Res. 2016;44(16):7527-7539.

52. Li Z, Guo J, Wu Y, Zhou Q. The BET bromodomain inhibitor JQ1 activates HIV latency through antagonizing Brd4 inhibition of Tat-transactivation. Nucleic Acids Res. 2013;41(1):277-287.

53. Bhagwat AS, Roe JS, Mok BY, Hohmann AF, Shi J, Vakoc CR. BET bromodomain inhibition releases the mediator complex from select cis-regulatory elements. Cell Rep. 2016;15(3):519-530.

54. Nielsen J, Christiansen J, Lykke-Andersen J, Johnsen AH, Wewer UM, Nielsen FC. A family of insulin-like growth factor II mRNA-binding proteins represses translation in late development. Mol Cell Biol. 1999;19(2):1262-1270.

55. Landthaler M, et al. Molecular characterization of human Argonaute-containing ribonucleoprotein complexes and their bound target mRNAs. RNA. 2008;14(12):2580-2596.

56. Elagib KE, et al. Jun blockade of erythropoiesis: role for repression of GATA-1 by HERP2. Mol Cell Biol. 2004;24(17):7779-7794.

57. Girard-Gagnepain A, et al. Baboon envelope pseudotyped LVs outperform VSV-G-LVs for gene transfer into early-cytokine-stimulated and resting HSCs. Blood. 2014;124(8):1221-1231.

58. Jain R, et al. RIP-Chip analysis: RNA-binding protein immunoprecipitation-microarray (Chip) profiling. Methods Mol Biol. 2011;703:247-263. 